\title{
CCN5 modulates the antiproliferative effect of heparin and regulates cell motility in vascular smooth muscle cells Andrew C Lake ${ }^{1}$ and John J Castellot Jr*2
}

\author{
Address: ${ }^{1}$ Program in Cell, Molecular and Developmental Biology, Sackler School of Biomedical Sciences, 136 Harrison Avenue, Tufts University, \\ Boston, MA 02111, USA and 2Department of Anatomy and Cell Biology, Tufts University School of Medicine, 136 Harrison Avenue, Boston, MA \\ 02111, USA \\ Email: Andrew C Lake - alakefrance@yahoo.com; John J Castellot* - john.castellot@tufts.edu \\ * Corresponding author
}

Published: 24 November 2003

Cell Communication and Signaling 2003, I:5

This article is available from: http://www.biosignaling.com/content/l/l/5

(C) 2003 Lake and Castellot; licensee BioMed Central Ltd. This is an Open Access article: verbatim copying and redistribution of this article are permitted in all media for any purpose, provided this notice is preserved along with the article's original URL.
Received: 15 September 2003

Accepted: 24 November 2003

\begin{abstract}
Background: Vascular smooth muscle cell (VSMC) hyperplasia plays an important role in both chronic and acute vascular pathologies including atherosclerosis and restenosis. Considerable work has focused on the mechanisms regulating VSMC proliferation and motility. Earlier work in our lab revealed a novel growth arrest-specific (gas) gene induced in VSMC exposed to the antiproliferative agent heparin. This gene is a member of the CCN family and has been given the name CCN5. The objective of the present study is to elucidate the function of CCN5 protein and to explore its mechanism of action in VSMC.
\end{abstract}

Results: Using RNA interference (RNAi), we first demonstrate that CCN5 is required for the antiproliferative effect of heparin in VSMC. We also use this gene knockdown approach to show that CCN5 is an important negative regulator of motility. To explore the mechanism of action of CCN5 on VSMC motility, we use RNAi to demonstrate that knock down of CCN5 up regulates expression of matrix metalloproteinase-2 (MMP-2), an important stimulator of motility in VSMC. In addition, forced expression of CCN5 via adenovirus results in reduced MMP-2 activity, this also corroborates the gene knock down results. Finally, we show that loss of CCN5 expression in VSMC causes changes in VSMC morphology and cytoskeletal organization, including a reduction in the amount and macromolecular assembly of smooth muscle cell $\alpha$-actin.

Conclusions: This work provides important new insights into the regulation of smooth muscle cell proliferation and motility by CCN5 and may aid the development of therapies for vascular diseases.

\section{Background}

Aberrant proliferation of smooth muscle cells (SMC) is the hallmark of several pathological states, including arteriosclerosis, persistent pulmonary hypertension in the newborn, pyloric stenosis, megaureter, and uterine fibroids. Because vascular SMC (VSMC) hyperplasia is responsible for the failure of a substantial fraction (as high as $30 \%$ ) of many vascular surgical procedures including percutaneous transluminal coronary angioplasty, coronary artery bypass grafts, arterio-venous 
shunts, endarterectomies, and heart transplants - considerable work has focused on the mechanisms regulating VSMC hyperproliferation and on the search for agents that can suppress VSMC mitogenesis. We and others have studied the glycosaminoglycan heparin, which inhibits VSMC proliferation and migration both in cell culture and in animal models [1]. We recently described and characterized a new member of the CCN family of growth factors [2], CCN5, that is induced and maintained by heparin treatment of VSMC and is expressed in a manner characteristic of a growth arrest specific (gas) gene [3]. We have also previously demonstrated that CCN5 is highly expressed in the aorta, and carotid artery, and is dynamically regulated upon vessel injury $[3,4]$.

The CCN family members are secreted, cell- and matrixassociated proteins that appear to play diverse and important roles in cell function [5-9]. They have been implicated in cell differentiation and survival, wound repair, vascular disease, fibrosis, angiogenesis, and tumorigenesis. Due to their expression patterns, interactions with cell surface proteins, and the ability to modulate cell functions, members of the $\mathrm{CCN}$ family have also been described as matricellular proteins [10]. All CCN proteins contain 38 conserved cysteine residues and share a homologous modular structure containing four distinct domains, with the exception of CCN5, which lacks the carboxy-terminal (CT) domain. Combined with its gas gene expression pattern, this observation led us to hypothesize that CCN5 plays a role in suppressing VSMC proliferation and motility.

The CCN5 gene and protein have been described by us and other laboratories and has been given a variety of names (HICP [11], rCop-1 [12], Cop-1 [3], Wisp-2 [13] and CTGF-L [14]). Homologues of CCN5 have been found in cells from mice, rats, and human sources [8,9]. Results from studies in our laboratory have recently demonstrated that overexpression of CCN5 protein has an antiproliferative and antimotility effect on VSMC [4]. Animal models also revealed that CCN5 is expressed in a manner consistent with control of smooth muscle cell growth in intact and injured arteries [4]. Intact carotid arteries show high levels of CCN5 expression in the media, while balloon-injured tissues display loss of CCN5 expression. Expression only returns upon resolution of the wound [4].

In the present study we examine the mechanism by which CCN5 affects VSMC behavior, and offer evidence that CCN5 mediates the antiproliferative effect of heparin on VSMC.

\section{Results}

RNAi knocks down CCN5 levels in growth arrested VSMC We have previously reported that overexpression of CCN5 in VSMC is able to inhibit proliferation and motility [4] suggesting that this protein plays an important role in the control of these processes. To more rigorously explore this possibility and to understand the mechanism of CCN5 in VSMC we employed RNA interference (RNAi) to knock down endogenous CCN5 expression in these cells. siRNA constructs designed to match nonconserved 21 nucleotide sequences within the CCN5 mRNA were transfected into VSMC. To determine the transfection efficiency of our siRNA we used fluorescently tagged siRNA. The results demonstrate that $>90 \%$ of the VSMC contain siRNA after transfection (Fig. 1A). Additionally, the level of fluorescent signal present in each individual cell does not significantly vary under our transfection conditions (Fig. 1A).

Real time quantitative PCR (Q-PCR) to detect levels of CCN5 message in CDNA derived from VSMC reveal that there is a significant decrease (77\%) of CCN5 expression in growth arrested cells treated with siRNA to target CCN5 message as compared to control siRNA treated cells (Fig. 1B). This decrease is similar to that seen in exponentially growing VSMC, which show an 80\% decrease in CCN5 levels when compared to untreated growth-arrested VSMC (Fig. 1B).

To corroborate the Q-PCR analysis we carried out a Western blot analysis to detect protein levels of CCN5 in lysates from growth-arrested VSMC. We demonstrate a significant (> 5-fold) decrease of CCN5 protein level in CCN5 siRNA treated cells as compared to either untreated or control siRNA treated cells (Fig. 1C). Taken together with the results from the Q-PCR, these data demonstrate that RNAi can efficiently and specifically reduce the amount of CCN5 protein in growth-arrested VSMC. Furthermore, they establish a valuable reagent and method for studying the requirement of CCN5 in VSMC function and for understanding its mechanism of action.

\section{Knock down of CCN5 does not induce VSMC apoptosis in VSMC}

It is possible that reduction of CCN5 levels in growtharrested VSMC could induce programmed cell death. To test if RNAi knock down of CCN5 in VSMC altered apoptosis, we measured caspase- 3 activity, as this enzyme is generally activated in cells undergoing apoptosis (Fig. 2). Caspase-3 activity was not elevated in lysates from growth-arrested VSMC as compared to the untreated or control siRNA treated cells (OD 0.035 vs. OD 0.017 and 0.035, Fig. 2). Positive and negative controls for caspase-3 activity were also included in the assay. Caspase- 3 activity was significantly elevated (OD 0.251) in lysates from positive control cells treated with staurosporine - a known 

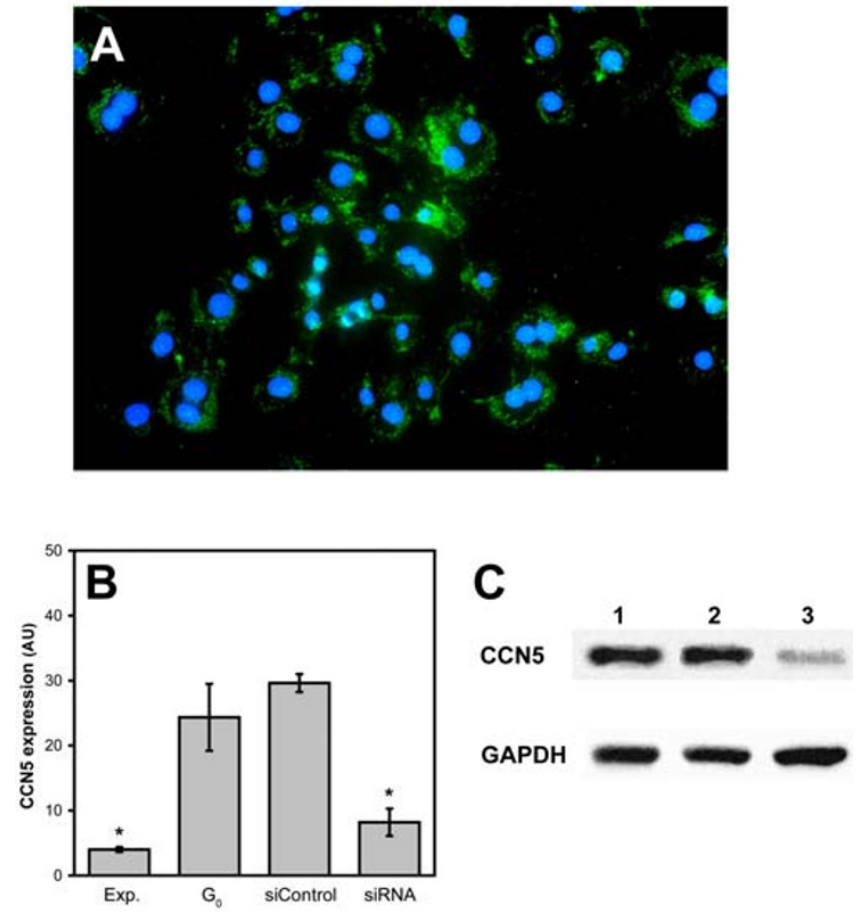

Figure I

Characterization of CCN5 RNAi in VSMC. A. VSMC transfected with fluorescein labeled siRNA and viewed live on an inverted fluorescent microscope to determine transfection efficiency. B. Q-PCR results: Total cellular RNA was isolated from control and CCN5 RNAi treated VSMC. After reverse transcription they were subjected to Q-PCR analysis to assay CCN5 message level. Graph is representative of relative CCN5 levels in the various conditions. * indicates $p<$ 0.05 with respect to the value for expression in growth arrested cells. (AU = Arbitrary Units) C. Western blots depicting reduced CCN5 protein expression in CCN5 siRNA treated VSMC. Top panel: Blot from cell lysates probed with anti-CCN5. Bottom Panel: Same blot stripped and reprobed with anti-GAPDH to insure equal loading. Lane I: untreated lysate Lane 2: control siRNA lysate Lane 3: CCN5 siRNA lysate.

inducer of VSMC apoptosis [15]. This activity is completely blocked (OD 0.012) by an inhibitor specific to caspase-3 (Fig. 2). These results establish that neither siRNA treatment nor knockdown of CCN5 in VSMC induces apoptosis in these cells.

\section{Reducing CCN5 levels decreases heparin sensitivity of VSMC}

Heparin has been previously shown to induce and maintain CCN5 expression in VSMC [3] and heparin also has a well-established antiproliferative effect on VSMC [16,17]. Given our previous results demonstrating that CCN5 can

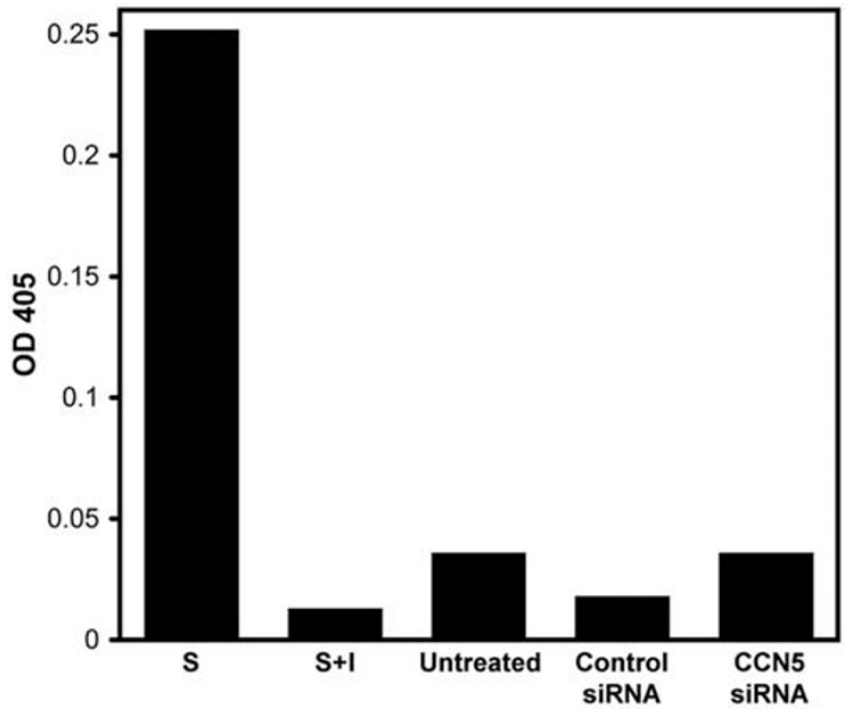

Figure 2

CCN5 siRNA does not induce apoptosis in VSMC. $2 \times$ $10^{6}$ VSMC treated with siRNA were collected after overnight incubation in 10\% FCS, washed in PBS, and then the lysates were assayed for caspase-3 activity. Exposure to staurosporine at $2.5 \mu \mathrm{M}$ in 10\% FCS RPMI overnight was used as a positive control for apoptosis. Caspase-3 inhibitor was also added as a control to show that measured OD is due to caspase-3 activity. Values have blank (OD 650 for reaction mix without substrate) subtracted. $\mathrm{S}=$ staurosporine, $\mathrm{S}+\mathrm{I}=$ staurosporine plus inhibitor.

abrogate VSMC proliferation [4] we thought it important to examine the role that CCN5 plays in the action of heparin on smooth muscle. VSMC treated with RNAi to knock down endogenous levels of CCN5 were tested for their sensitivity to the antiproliferative effect of heparin.

Proliferation assays were performed on VSMC using a range of heparin doses $(10-300 \mu \mathrm{g} / \mathrm{ml})$. The cells tested were either untreated or transfected with siRNA. The control siRNA treated VSMC show no change in the $50 \%$ effective dose $\left(E_{50}\right)$ of heparin inhibition $(\sim 60 \mu \mathrm{g} / \mathrm{ml}$, Fig. 3) compared to the untreated cells $(\sim 60 \mu \mathrm{g} / \mathrm{ml}$, Fig. 3$)$. The $\mathrm{ED}_{50}$ for heparin inhibition of VSMC treated with CCN5 siRNA is $\sim 5$ fold greater than both controls $(\sim 300$ $\mu \mathrm{g} / \mathrm{ml}$, Fig. 3). By using RNAi to knock down CCN5 we have demonstrated that this protein can alter the antiproliferative effect of heparin in VSMC. This result also provides a potentially important link between the presence of CCN5 in VSMC, and the antiproliferative effect of heparin. 


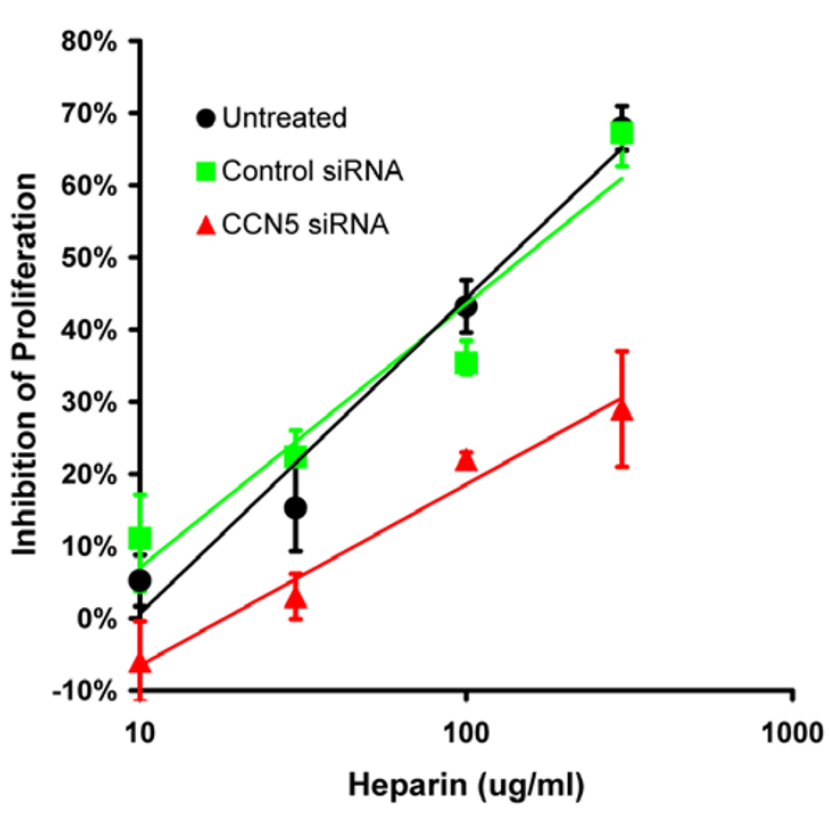

Figure 3

CCN5 RNAi reduces heparin sensitivity of VSMC. $8 \times$ $10^{3}$ VSMC/well, previously untreated or treated with control siRNA or CCN5 siRNA, were washed with RPMI and placed in RPMI containing $0.4 \%$ FCS for $72 \mathrm{~h}$. Cells were released from quiescence by replacing the low serum medium with normal growth medium, RPMI-I640 containing I0\% FCS. A range of heparin doses was added to the medium ( $10-300$ $\mu \mathrm{g} / \mathrm{ml})$. After 4 days of growth, cell proliferation was determined by direct cell counting using a Coulter Counter. The net growth of VSMC was obtained by subtracting the starting cell number from the final count results (at the time of release from $\mathrm{G}_{0}$ ).

\section{Knock down of CCN5 increases motility of VSMC}

VSMC migration from the media into the intimal surface of the blood vessel is an important step during restenosis following vascular surgical procedures. We have already established that CCN5 overexpression can significantly reduce motility of VSMC [4]. To ascertain whether CCN5 controls basal cell locomotion during $\mathrm{G}_{0}$ we have used RNAi to knock down levels of the protein present in growth arrested VSMC and observed the effect on cellular motility and invasiveness in two independent assays.

Monolayer scratch wound assays have been used by our laboratory and others to study VSMC motility $[4,18,19]$. In the case of the experiments presented here, the near confluent VSMC were treated with siRNA, growth arrested, and then wounded. The cells were kept in a state of serum deprivation for the duration of the experiment. The results therefore measure basal motility of the VSMC in the normally non-motile state. We observed almost no movement of cells into the wounded area in both untreated and control siRNA treated monolayers (4 cells and 6 cells respectively, Fig. 4A,4B), while CCN5 knock down greatly increased wound coverage (123 cells, Fig. 4C).

VSMC in vivo must pass though the basement membrane of the endothelial cell (EC) layer to enter the intimal surface of the artery wall. To mimic the in vivo process and to independently corroborate the scratch wound assay results, we employed transwell chambers coated with GFR-Matrigel, which may be thought of as a partially reconstituted basement membrane. This method has been used previously by our laboratory to show that CCN5 overexpression reduces the ability of VSMC to migrate through a matrix environment [4]. Using this approach, we now demonstrate that decreasing CCN5 levels in growth-arrested VSMC can dramatically increase their motility in response to a chemoattractant (10\% FCS, Fig. $5)$. The filters from the untreated and control siRNA conditions (Fig. 5A,5B) show significantly fewer cells have entered the Matrigel and crossed the filter compared to the CCN5 siRNA treated cells (Fig. 5C). VSMC with reduced CCN5 levels (18.5 cells per field, Fig. 5D) exhibited a twofold increase in motility stimulated by $10 \%$ FCS as compared to untreated and control VSMC ( 9 and 7.8 cells per field respectively, Fig. 5D).

\section{CCN5 regulates VSMC matrix metalloproteinase (MMP) expression}

Taken together, the results of the scratch wound experiment and the transwell assay just described demonstrate that CCN5 is capable of regulating VSMC motility. The following experiments were designed to explore the mechanism of action of CCN5 on VSMC motility. We examine the role of ECM modification, as well as cytoskeletal reorganization as potential downstream effectors of CCN5.

Matrix modification is a well-established requirement for increased motility of VSMC and other cell types. For example, MMPs are known to play an important role in VSMC migration in vivo, contributing to arteriosclerosis, and restenosis after balloon injury [20]. In addition, it has already been shown that MMP-2 and 9 are required for migration of VSMC through Matrigel in vitro [21,22]. To determine whether or not the presence of CCN5 could influence the expression of MMP-2 in VSMC we have induced overexpression of CCN5 via adenovirus and also knocked down CCN5 using RNAi. We then employed gelatin zymography to measure the level of MMP-2 released by VSMC under various conditions.

Overexpression of CCN5, via adenovirus, reduces MMP-2 levels in conditioned medium taken from PDGF-BB 
Untreated

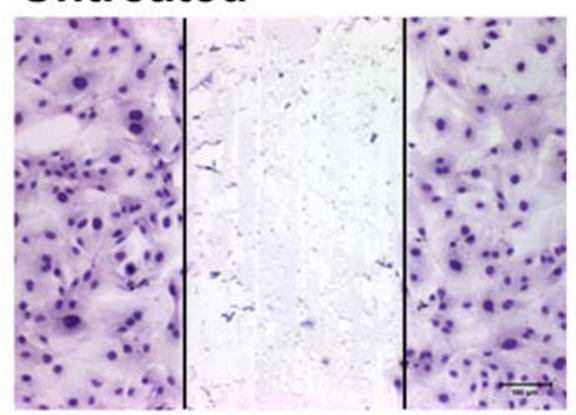

4
Control siRNA

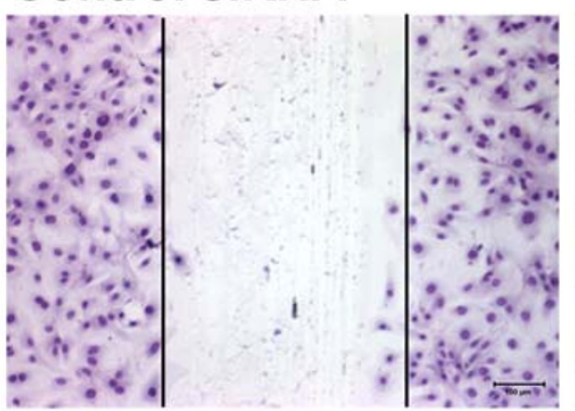

6
CCN5 SiRNA

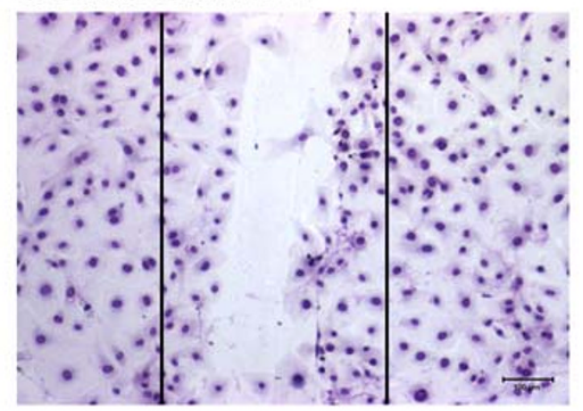

123

\section{Figure 4}

CCN5 targeted siRNA promotes motility in growth arrested VSMC. VSMC were plated at near confluence on glass chamber slides and then transfected overnight with $600 \mathrm{ng}$ of siRNA to knockdown CCN5 levels or transfected with control siRNA. Following transfection VSMC were growth arrested by serum starvation for 72 hours. The confluent monolayers were then scratched uniformly with a pipette tip, excess cells were washed away, and serum free growth medium was added back. Initial pictures of the scratches were taken for reference (demarcation of initial wounds is represented by the white lines in each panel). The monolayers were then incubated for 48 hours at $37^{\circ} \mathrm{C}$, fixed, stained with Diff-Quik to visualize cells and the extent of cell movement into the wound was measured by counting nuclei. The numbers below the panels represent the number of cells that have migrated into the wound area.

stimulated VSMC (Fig. 6). While adenovirus infection itself raises the level of total MMP-2 activity in growth arrested cells (lane 1 vs. lane 4, Fig. 6), PDGF-BB raises total MMP-2 levels in both controls (untreated and GFP control infected) in a dose dependant manner (lanes 2-3 vs. lanes 5-6, Fig. 6). Conditioned medium from growth arrested VSMC overexpressing CCN5 has the same amount of total MMP-2 as GFP control conditioned medium (lane 7 vs. lane 4, Fig. 6). Conditioned medium from the CCN5 adenovirus infected cells that were treated with PDGF-BB (lanes 8-9, Fig. 6) has approximately 3fold less total MMP-2 then GFP control conditioned medium (lanes 5-6, Fig. 6).

Conversely, gelatin zymography indicates that knock down of CCN5 in growth arrested VSMC increases the level of MMP-2 produced (Fig. 7). Conditioned medium from untreated (lane 1, Fig. 7) and control siRNA treated cell (lane 2, Fig. 7) contain the same amount of total MMP-2. In contrast, conditioned medium from CCN5 siRNA treated cells contain a 2-fold increase in total MMP2 (lane 3, Fig. 7) produced by the growth arrested VSMC. This observation, combined with the forced expression data, indicates that levels of CCN5 present in VSMC can modulate the amount of MMP-2 produced. High CCN5 levels down-regulate MMP-2 production, whereas low levels of CCN5 in VSMC permit up-regulation of MMP-2.

\section{Knock down of CCN5 alters VSMC morphology}

Confluent monolayers of growth-arrested VSMC display the classic "hill and valley" morphology, which is characterized by intertwined and overlapping bipolar cells (Fig. $4 \mathrm{~A}$ and $8 \mathrm{~A}$ ). Treatment with control siRNA does not significantly change this morphology (Fig. 4B and $8 \mathrm{~B}$ ), while CCN5 knock down does (Fig. 4C and 8C). CCN5 siRNA treated cells have a spread epithelial-like appearance in contrast to that of untreated VSMC (Fig. 8C). This phenomenon could explain, at least in part, the results we observed in the scratch wound assay, and is also suggestive of changes in VSMC cytoskeletal arrangement.

\section{CCN5 may regulate the $\alpha$-actin cytoskeleton}

Growth arrested VSMC in culture normally produce relatively high amounts of smooth muscle $\alpha$-actin arranged in a well-organized cytoskeleton (Fig 9A). It is possible that CCN5 could be involved in the maintenance of stable $\alpha$ actin cytoskeleton in VSMC. Changes in cytoskeletal organization could also account for the changes in cellular motility and morphology we observe when CCN5 is knocked down with RNAi. We therefore examined $\alpha$-actin location (Fig. 9) and mRNA expression (Fig. 10) in growth-arrested, siRNA treated VSMC to determine if reduced CCN5 levels could affect changes in cytoskeletal arrangement and/or production. The results demonstrate that CCN5 siRNA treated VSMC lack the prominent wellorganized stress fibers observed in untreated VSMC (Fig. $9 A, 9 B)$. Q-PCR analysis of $\alpha$-actin message expression in 

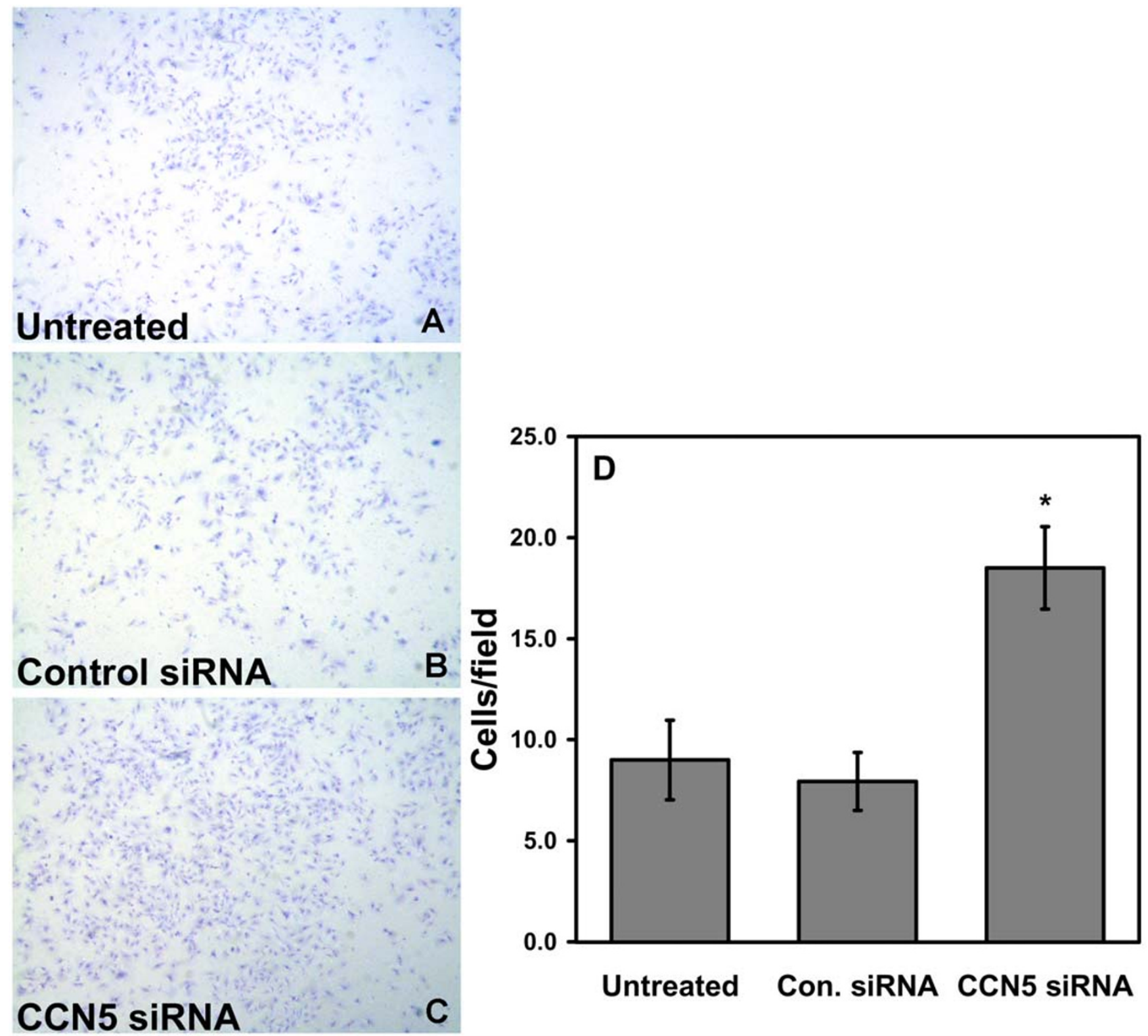

\section{Figure 5}

VSMC GFR-Matrigel transwell assay. VSMC previously untreated or treated with control or CCN5 siRNA $\left(3 \times 10^{4}\right.$ cells per well in $500 \mu \mathrm{l}$ ) were plated into the upper portion of GFR-Matrigel chambers (BD Biosciences) and allowed to invade for 30 hours in response to $10 \%$ FCS. Filters were cleaned, fixed, stained, and counted as described in Experimental Procedures. Pictures are of representative stained filters photographed at low power under a dissecting microscope $\mathbf{A}$. Untreated VSMC B. Control siRNA treated VSMC C. CCN5 siRNA treated VSMC. D. Graph representing quantitation of the results from 4 fields on triplicate filters. * indicates $p<0.05$ with respect to the value for untreated VSMC. 


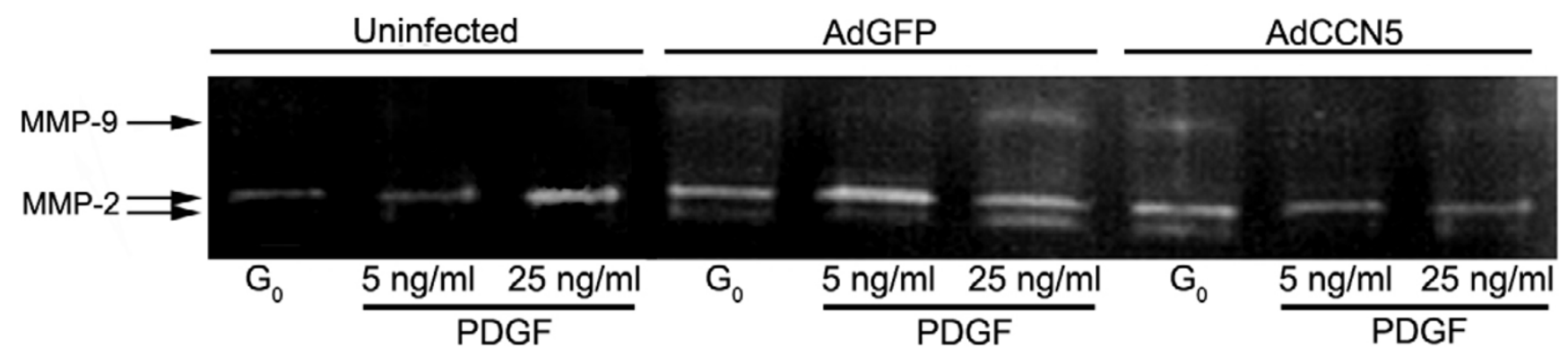

Figure 6

PDGF-induced MMP production is reduced in AdCCN5-infected cells. Uninfected VSMC or VSMC infected with AdCCN5 or AdGFP were growth arrested by serum starvation for $72 \mathrm{~h}$, and then treated overnight with serum-free medium containing the indicated concentrations of PDGF-AB. Conditioned media were collected, concentrated, and run on an SDSPAGE gelatin zymogram. The gel was stained with Coomassie Blue. The bands lacking stain indicate MMP activity. UPper band is MMP9 (Gelatinase B); lower bands are MMP2 (Gelatinase A) in both active (lower) and inactive (upper) forms as shown. Ad = Adenovirus.

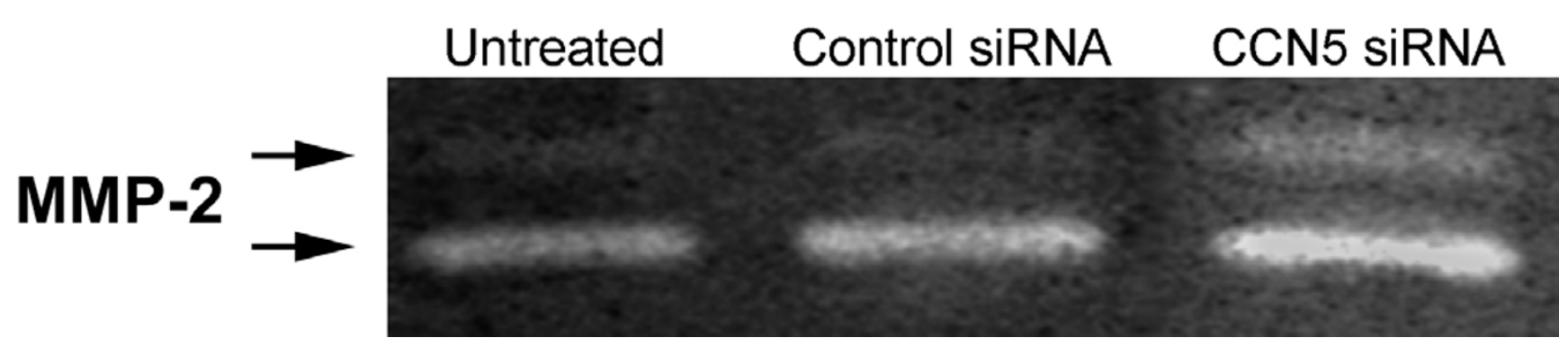

Figure 7

CCN5 knock down increases MMP-2 expression in growth-arrested VSMC. Untreated, control siRNA, or CCN5 siRNA treated VSMC were growth arrested by serum starvation for $72 \mathrm{hr}$. Conditioned media were collected, concentrated, and run on an SDS-PAGE gelatin zymogram. The gel was stained with Coomassie Blue. The bands lacking stain indicate MMP activity. Bands are MMP2 (Gelatinase A) in both active (lower) and inactive (upper) forms as shown.
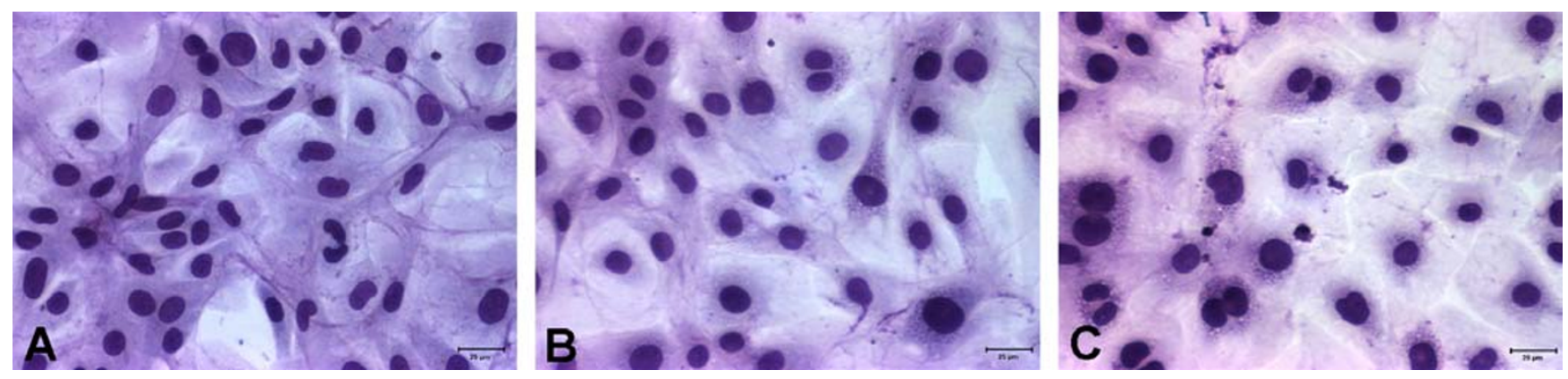

Figure 8

CCN5 knock down alters VSMC morphology. Untreated, control siRNA, or CCN5 siRNA treated VSMC were growth arrested by serum starvation for $72 \mathrm{hr}$. Cells were then placed in serum free RPMI 1640 medium for 48 hours, fixed and stained with Diff-Quik to visualize morphology. A. Untreated B. Control siRNA treated C. CCN5 siRNA treated. 

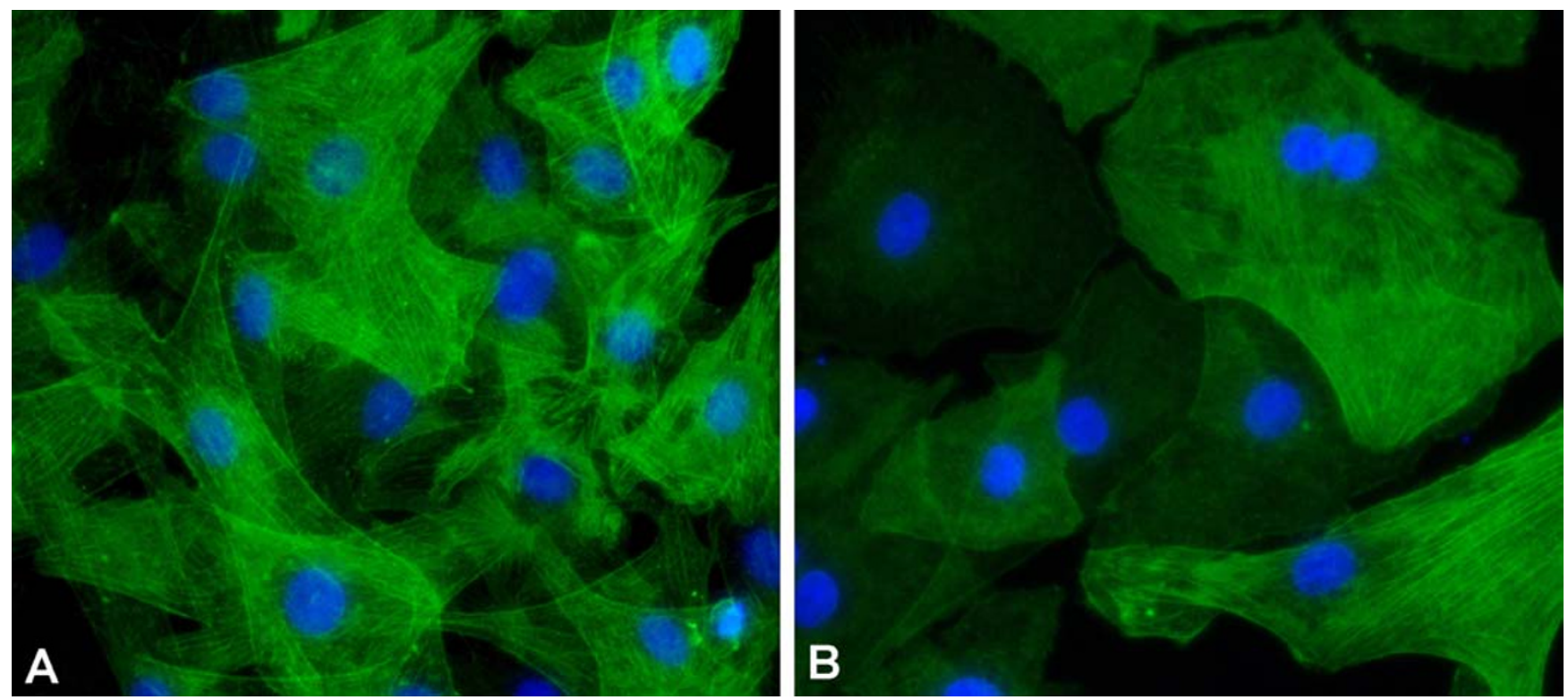

Figure 9

CCN5 knockdown alters $\alpha$-actin cytoskeleton. Untreated or CCN5 siRNA treated VSMC were growth arrested by serum starvation for $72 \mathrm{hr}$. Cells were then fixed and stained for $\alpha$-actin (anti- $\alpha$-actin mouse monoclonal antibody), secondary (Alexa-488-conjugated anti-mouse antibody; green), and nuclei (Hoechst stain; blue). A. Untreated VSMC. B. CCN5 siRNA treated VSMC

these cells indicates that CCN5 siRNA treated cells have only half as much $\alpha$-actin mRNA (0.9, Fig. 10) when compared to untreated and control siRNA-treated VSMC (1.7 and 1.8, Fig. 10). Both of these results suggest that CCN5 is important to normal production and arrangement of VSMC $\alpha$-actin cytoskeleton.

\section{Conclusions}

In this study we report several novel observations on the functions and possible mechanism of action of CCN5, a member of the CCN family of matricellular proteins. We demonstrate that CCN5 is necessary for the antiproliferative effect of heparin on VSMC in culture (Fig. 3). We provide functional evidence that CCN5 plays an important role in the control of VSMC motility (Fig. 4, 5). Finally, we offer insight into the mechanism of action of CCN5 on VSMC by showing that this protein can control the expression of MMP-2 (Fig. 6, 7) and may also play an important role in control of cell morphology (Fig. 8) and the organization of the actin cytoskeleton (Fig. 9, 10).

To examine the requirement for CCN5 protein in cell function, we have established the use of RNAi to significantly (5-fold) knock down expression of this protein in growth-arrested VSMC (Fig. 1). Our group has previously established CCN5 as a gas gene in these cells [3]. VSMC in vivo also maintain a quiescent state and express high levels of CCN5 [4]. Importantly, by using the RNAi technique, levels of the protein can be lowered in growth-arrested cells to the level observed in exponentially growing VSMC. We have used this tool to understand the requirement for CCN5 in maintaining the characteristics of growth arrested VSMC, such as sensitivity to heparin's antiproliferative effect, low rate of motility, and a well organized actin cytoskeleton.

Our data provides strong evidence that CCN5 mediates, at least in part, the antiproliferative effect of heparin on VSMC (Fig. 3). The negative effect of heparin on VSMC mitogenesis has been known for more than two decades; however, the mechanism of action is still unclear. While it is likely that heparin has multiple modes of action in VSMC, induction of CCN5 appears to be a major contributor to its antiproliferative effect.

Using two independent approaches, we have shown that knock down of CCN5 levels in growth-arrested VSMC leads to increased motility (Fig. 4,5). We had previously shown that the opposite is true for CCN5 overexpression in VSMC [4]. Taken together these results demonstrate that CCN5 is a negative regulator of VSMC motility and invasiveness. Since we already know that relatively high levels of CCN5 are present in quiescent VSMC residing in the arterial media in vivo $[3,4]$, it is tempting to speculate 


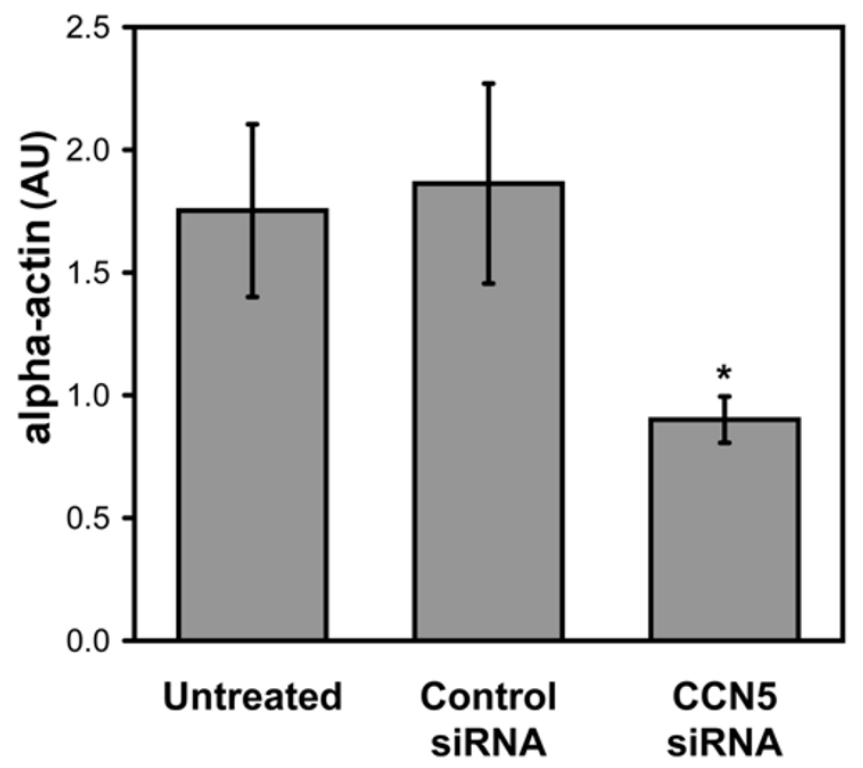

Figure 10

CCN5 knockdown reduces VSMC $\alpha$-actin expression. Total cellular RNA was isolated from control and CCN5 RNAi treated VSMC. After reverse transcription they were subjected to Q-PCR analysis to assay $\alpha$-actin message level. Graph is representative of relative $\alpha$-actin levels in the 3 conditions. * indicates $p<0.05$ with respect to the value for untreated VSMC. (AU = Arbitrary Units)

that CCN5 could be responsible, at least in part, for maintaining their non-motile, non-proliferative state. Lending support to this hypothesis, results from the current study demonstrate that the expression of MMP-2 in VSMC is inversely correlated with expression of CCN5 (Fig. 6, 7). MMPs are necessary for VSMC invasion during in vitro assays $[23,24]$, and MMP-2 is known to play an important role in atherosclerotic plaque formation and restenosis after vessel injury [25-28]. Interestingly, another family member, CCN2, which is known to have a pro-motility effect on VSMC [29], has also been shown to increase MMP-2 expression in VSMC [30] and decrease levels of tissue inhibitor of MMP-1 (TIMP-1) [31]. This is especially intriguing because CCN2 is not found at high levels in healthy aorta, but is up regulated 50-100 fold in atherosclerotic blood vessels [32]. In contrast, we observe high levels of CCN5 in healthy rat arterial tissue $[3,4]$ and expression of CCN5 is abolished during restenosis following balloon injury [4].

Our results suggest that CCN5 may play a role in the control of VSMC morphology and regulation of the cytoskeleton (Fig. 8, 9, 10). These changes in morphology and cytoskeletal arrangement are consistent with the modula- tion of motility observed. Indirect evidence demonstrates that other CCN proteins possess the ability to alter cytoskeleton of cells. Another CCN family member, CCN2, was recently shown to influence cytoskeletal arrangement in mesangial cells, [33] a cell type closely related to VSMC. CCN1 and CCN2 both appear to act through integrins [7]. These transmembrane receptors are responsible for ECM binding on the outside and make connections with the actin cytoskeleton in the cytoplasm [34]. Integrins are central to the formation of focal adhesion complexes, which are an integral part of stress fiber formation within cells [34]. It is tempting to speculate that CCN5 could help to stabilize these complexes, and experiments to test this idea are underway. As discussed above, these CCN family member proteins are both known to stimulate motility in various cell types including VSMC [8]. Additionally, CCN4, a protein capable of inhibiting invasion and motility of tumor cells both in vivo as well as in vitro, inhibits Rac activation [35], which is thought to be intimately involved in regulation of cytoskeletal organization [35].

In this study we provide functional data that CCN5 is capable of inhibiting processes in cultured VSMC that are thought to be important in the pathogenesis of arteriosclerosis and restenosis: motility and matrix modification. We also provide evidence that CCN5 mediates the antiproliferative effect of heparin on VSMC. We also provide data suggesting that CCN5 is important for the maintenance of normal VSMC morphology and $\alpha$-actin expression. While it is clear that the biological functions as well as the pathophysiologic roles, if any, for CCN5 are complex and likely to be tissue-specific, these data, taken together with results from our previous studies [4], suggest a role for CCN5 in the control of VSMC motility and proliferation in both the healthy and injured artery wall. Experiments to examine this possibility are currently underway and should help shed light on the role of CCN5 in VSMC in vivo.

\section{Methods \\ Materials}

All tissue culture plastic ware, including growth factor reduced (GFR)-Matrigel chambers, were obtained from BD Biosciences (Lincoln Park, NJ). $8 \mu \mathrm{m}$ polycarbonate filter membranes for use in modified Boyden assays were purchased from NeuroProbe Inc. (Cabin John, MD). Premium fetal calf serum (FCS) was purchased from HyClone (Logan, UT). RPMI base media, trypsin-EDTA, glutamine, and penicillin-streptomycin were purchased from Gibco Life Technologies (Grand Island, NY). Heparin, obtained from Glycomed (Alameda, CA) was derived from porcine mucosa (sodium salt; average molecular mass, $15 \mathrm{kDa}$ ). Pre-cast SDS-gels and running system were obtained from Bio-Rad (Hercules, CA). ECL 
Renaissance kit was purchased from New England Nuclear (Boston, MA). Anti-CCN5 affinity-purified rabbit polyclonal antibody was obtained from FibroGen (San Francisco, CA). HRP and alexa-488-conjugated anti-rabbit secondary antibodies were purchased from Jackson ImmunoResearch Laboratories Inc. (West Grove, PA) and Molecular Probes (Eugene, OR) respectively. All other chemicals and reagents were purchased from Sigma (St. Louis, MO).

\section{Cell Culture}

Primary cell cultures

Aortic muscle cells (VSMC) were obtained from aortae of Sprague-Dawley rats (Charles River Breeding Laboratories, Inc. Wilmington, MA). They were isolated, cultured, and characterized as previously described [36]. Briefly, the abdominal segment of the aorta was removed and the fascia cleaned away under a dissecting microscope. The aorta was cut longitudinally, and small pieces of the media were carefully stripped from the vessel wall. Two or three such strips were placed in $60 \mathrm{~mm}$ dishes. Within 1-2 weeks, VSMC migrated from the explants; they were capable of being passaged approximately 1 week after the first appearance of cells. They were identified as VSMC by their characteristic hill-and-valley growth pattern and indirect immunofluorescence staining for VSMC specific $\alpha$-actin. All cultures were maintained in RPMI-1640 medium containing $10 \%$ FCS (10\% FCS/RPMI) at $37^{\circ} \mathrm{C}$ in a humidified, 5\% $\mathrm{CO}_{2} / 95 \%$ air atmosphere.

\section{Growth arrest of cells}

Cells were routinely plated at various densities, washed with serum-free RPMI and placed in 0.4\% FCS/RPMI for $72 \mathrm{~h}$. Flow cytometry and determination of $\left[{ }^{3} \mathrm{H}\right]$ thymidine-labeled nuclei indicated that greater than $95 \%$ of the cells were arrested in $G_{0}\left(G_{1}\right)$ [37]. Cells were released from quiescence by replacing the low serum medium with $10 \%$ FCS/RPMI. Cultures were used at passage 8 or lower.

\section{RNAi production and Transfection Production of siRNA}

CCN5 targeted 21 nucleotide siRNA was produced using the Silencer siRNA in vitro transcription kit (Ambion, Austin, TX). The targeted sequence resides within the open reading frame of the CCN5 mRNA, 306 nucleotides downstream of the start codon. The method of Tuschl et. al. [38] was used to determine the target sequence. The sequence is as follows: aatggccgcaggtacctggat GAPDH targeted siRNA, was also produced for use as a positive control for message knockdown, and also for transfection condition optimization. Negative control siRNA, a 21nucleotide RNA duplex with no known sequence homology, was also purchased from Ambion.

\section{Production of fluorescent-tagged siRNA}

Fluorescein labeled siRNA used to detect transfection efficiency was produced with the Silencer Fluorescein siRNA Labeling Kit from Ambion (Austin, TX). Negative control siRNA was labeled using the kit. This siRNA was then transfected into VSMC as described below. Cells were then visualized and digital images were obtained with a RT Color Spot camera (Diagnostic Instruments Inc.) on a Nikon inverted fluorescence microscope. Figures were prepared with Adobe Photoshop v. 6.0.

\section{Transfection of siRNA}

Transfection of siRNA into VSMC was achieved by using the GeneSilencer siRNA Transfection Reagent, produced by Gene Therapy Systems (San Diego, California). Briefly, VSMC were plated overnight to achieve 50-70\% confluent monolayers. siRNA and transfection reagent complex was added to the cells in $10 \%$ serum containing medium and incubated 24 hours at $37^{\circ} \mathrm{C}$. Cells were then washed with $10 \%$ serum containing medium for 1 hour and then growth arrested for 72 hours as described above.

\section{RNA analysis}

mRNA collection and reverse transcription

Total cellular RNA from the various conditions described above was harvested from $100 \mathrm{~mm}$ culture dishes as follows. Monolayers were rinsed twice with phosphate buffered saline (PBS, pH 7.6) and RNA was collected using the RNeasy Mini kit (QIAGEN, Valencia, CA). Contaminating DNA was removed using the DNA-free kit (Ambion, Austin, TX), and reverse transcription was performed using the RETROscript kit (Ambion, Austin, TX). All assays were performed according to the manufacturer's protocol. Controls containing no reverse transcriptase were used to check for genomic DNA contamination in each sample. PCR using the HotStarTaq kit (QIAGEN, Valencia, CA) and examination of products on a $1.8 \%$ agarose gel confirmed the absence of genomic DNA.

\section{Real time Quantitative PCR}

Primers were purchased from IDT (Coralville, IA). The sense CCN5 (GenBank accession number gi 7739780) primer was 5'-CACCAACTTTCTGCCCTTGT-3' (position 796-815), and the antisense CCN5 primer was 5'-ATCTCCAGTTGGCAGAATCG-3' (position 922-941). These primers produced a product size of 146 . The sense GAPDH (GenBank accession number gi 8393417) primer was 5'-GAAGGGCTCATGACCACAGT-3' (position 538557), and the antisense GAPDH primer was 5'-GGATGCAGGGATGATGTTCT-3' (position 635-654), producing a product size of 117 . Real-time PCR was performed using SYBR Green PCR Master Mix (Applied Biosystems, Foster City, CA). The reactions were performed on the GeneAmp 5700 Sequence Detection System (PE Applied Biosystems, Foster City, CA). No template and no reverse 
transcriptase samples were used as controls. The cycling conditions were: $95^{\circ} \mathrm{C} 10 \mathrm{~min}$, and 40 cycles of $95^{\circ} \mathrm{C} 15$ $\mathrm{s}$ and $60^{\circ} \mathrm{C} 1 \mathrm{~min}$. The standard dissociation protocol was performed to confirm the absence of primer dimers, and product size was determined by running PCR products on a $1.8 \%$ agarose gel. A standard curve $\left(\mathrm{C}_{t}\right.$ vs. $\left.\log \mathrm{C}_{0}\right)$ was constructed using a dilution series of rat lung total RNA (Ambion, Austin, TX) transcribed to cDNA using the same protocol outlined above, and relative amounts of CCN5 and GAPDH were determined.

\section{Adenovirus production and infection}

An adenovirus expressing both Green Fluorescent Protein (GFP) and CCN5 tagged by a nine amino acid HA epitope on the C-terminus, was produced using the Ad-Easy system provided and described by He et al. (1998). This virus has been described in more detail previously [4]

VSMC are routinely infected in our laboratory by the following method. Cells are first washed and exposed to various amounts of virus for 2 hours in serum free RPMI1640 with occasional agitation. 10\% FCS/RPMI containing medium is then added back to the cells. After 1-2 days infected cells can be trypsinized and used for experiments as usual. Infection can be monitored quickly by observing the fraction of cells expressing GFP in the population. It has been previously demonstrated in our lab that the AdCCN5 used induces overexpression of CCN5 adjustable over a range of moi [4].

\section{Protein analysis}

Western Blotting

Proteins from the various conditions described above were harvested from $100 \mathrm{~mm}$ culture dishes as follows, with all procedures performed at $4{ }^{\circ} \mathrm{C}$. Cells were rinsed twice with cold TBS (20 mmol/L Tris, pH 7.6, $137 \mathrm{mmol} /$ $\mathrm{L} \mathrm{NaCl}$ ) and lysed with $100 \mu \mathrm{l}$ of RIPA lysis buffer. Lysates were transferred to Eppendorf tubes, rocked for $20 \mathrm{~min}$ and spun at $12,000 \mathrm{rpm}$ for $10 \mathrm{~min}$ in an Eppendorf microfuge (Hamburg, Germany). Supernatant was stored at negative $20^{\circ} \mathrm{C}$ until use. Protein estimations were performed using the Pierce BCA method adapted for microtiter plates. Extracts containing from $10-40 \mu \mathrm{g}$ of protein were boiled in $2 \mathrm{X}$ SDS sample loading buffer, resolved by SDS-PAGE (either $10 \%$ or $4 \rightarrow 20 \%$ gradient gels were used), and blotted onto $0.2 \mu \mathrm{m}$ pore size Immun-Blot PVDF membranes (Bio-Rad, Hercules, CA) in Towbin buffer without methanol (25 mmol/L Trizma base, 192 $\mathrm{mmol} / \mathrm{L}$ glycine) at $200 \mathrm{~mA}$ for five $\mathrm{h}$. The blots were dried and rewetted. Membranes were blocked for $1 \mathrm{~h}$ in TBS containing 5\% milk and Western blots were performed using primary antibodies described above (1:500) and HRP-conjugated anti-rat or anti-rabbit IgG $(1: 10,000)$ in TBST (TBS $+0.2 \%$ Tween 20). Bands were visualized using the NEN (Boston, MA) Renaissance enhanced chemiluminescence (ECL) detection reagents and autoradiography as described by the vendor. Prestained protein standard markers (BioRad) were used as molecular weight markers. Densitometry analysis of films was performed using the Stratagene (La Jolla, CA) Eagle Eye II system. Blots were stained with Amido Black stain to confirm equal loading and proper transfer of proteins to the membrane.

\section{Immunocytochemistry}

VSMC were plated onto $18 \mathrm{~mm}$ cover slips in 24-well dishes at a density of $8 \times 10^{3}$ cells/well. Cells were growth arrested and subsequently reintroduced to normal growth medium for various times. Cover slips were then fixed and blocked as described above, followed by exposure to the affinity-purified rabbit anti-peptide antibody specific to CCN5 or a mouse monoclonal antibody specific to smooth muscle $\alpha$-actin. Secondary antibody, goat antimouse conjugated to alexa-488 (Molecular Probes, Eugene, OR) was used to visualize smooth muscle $\alpha$-actin in the cells. Hoechst 33258 stain was also added to the mounting medium in order to visualize cell nuclei. Digital images were obtained with a RT Color Spot camera (Diagnostic Instruments Inc.) on a Nikon inverted fluorescence microscope. Figures were prepared with Adobe Photoshop v. 6.0.

\section{Gelatin Zymography}

Conditioned medium from growth arrested cells was loaded into in precast $10 \%$ acrylamide gels impregnated with gelatin (Biorad, Hercules, CA) and subjected to SDSPAGE electrophoresis. Gels were then renatured $(2.5 \%$ Triton X-100), developed overnight (50 mM Tris, $\mathrm{pH} 7.6$, $\left.10 \mathrm{mM} \mathrm{CaCl}_{2}\right)$, and stained with Commassie blue for 1 hour (0.5\% Coomassie blue, 30\% isopropanol, $10 \%$ acetic acid). After destaining (10\% isopropanol 1 hour followed by water to allow gel to swell to normal size), gels were photographed using the Stratagene (La Jolla, CA) Eagle Eye II system.

\section{Cell proliferation assay}

Cell proliferation assays were performed as described previously [39]. Briefly, $8 \times 10^{3}$ cells were plated into $16 \mathrm{~mm}$ multiwell dishes. In some cases, cells were previously transfected with siRNA as described above. Cells were then growth arrested as above. Cultures were released from $\mathrm{G}_{0}$ by exposure to $10 \% \mathrm{FCS} / \mathrm{RPMI}$. In some cases cells were also treated with $10 \%$ FCS/RPMI containing a range of $10-300 \mu \mathrm{g} / \mathrm{ml}$ heparin. Cells were allowed to grow for the indicated time and then counted in duplicate after trypsinization using a Coulter counter (Fullerton, CA). The net growth of VSMC was obtained by subtracting the starting cell number from the above count results (at the time of release from $\mathrm{G}_{0}$ ). Experimental conditions 
were performed in triplicate on two separate occasions. Degree of inhibition is determined as follows:

percent inhibition $=1-\frac{\text { net proliferation in experimental well }}{\text { net proliferation in FCS control well }} \times 100$

\section{Cell motility}

Scratch wound motility assay:[18,19]

siRNA transfected VSMC were plated at confluence onto glass chamber slides. The following day a uniform straight scratch was made in the monolayer using a $200 \mu \mathrm{l}$ yellow plastic pipet tip (Fisher). Monolayers were washed gently, marked (for reference) and photographed using Nomarski optics on an inverted microscope. After incubation for $30 \mathrm{~h}$ at $37^{\circ} \mathrm{C}$, the cells were fixed in $1 \%$ paraformaldehyde and mounted. Cells were stained using the Diff-Quik stain set (Dade Behring Inc., Newark, DE). Calculations are based on the number of nuclei that have moved into the wound area. Four counts were made at various points along each wound that were photographed initially and marked. Cell numbers are derived from the average of these fields from triplicate samples.

GFR-Matrigel transwell chamber assay:[2I,25,28]

VSMC motility through matrix was measured using chambers (BD Biosciences, New Bedford, MA) containing an 8 $\mu \mathrm{m}$ pore size polycarbonate filter insert that divides the chamber into upper and lower portions. The filters were either uncoated or uniformly coated with GFR-Matrigel. We used the protocol provided by the supplier. Briefly, 3 $\times 10^{4}$ cells were added to the upper chamber of the insert while 10\% FCS was added in RPMI-1640 to the lower chambers. After allowing the assay to proceed for $32 \mathrm{~h}$, cells on the top of the filter were removed by gentle swabbing and the remaining cells on the bottom side of the filter were stained using Diff-Quik (Dade Behring Inc., Newark, DE), and counted under a light microscope with an eyepiece grid to visualize set fields. At least 4 fields in triplicate wells were counted for each condition.

\section{Detection of apoptosis}

The ApoAlert Caspase-3 Colorimetric Assay Kit by Clontech (Palo Alto, CA) was used according to the manufacturer's instructions to determine the amount of apoptosis in VSMC cultures. Briefly, $2 \times 10^{6}$ AdGFP-infected, AdCCN5-infected, or uninfected control cells were collected after overnight incubation in $10 \%$ FCS, washed in PBS, and then the lysates were assayed for caspase- 3 activity. Exposure to staurosporine at $2.5 \mu \mathrm{M}$ in $10 \%$ FCS/ RPMI overnight was used as a positive control for apoptosis [15].

\section{List of abbreviations}

$\mathrm{CCN}=$ cysteine-rich61/connective tissue growth factor / nephroblastoma overexpressed
$\mathrm{ECM}=$ Extra cellular matrix

FCS $=$ fetal calf serum

gas $=$ growth arrest-specific

$\mathrm{GFP}=$ green fluorescent protein

$\mathrm{MMP}=$ matrix metalloproteinases

moi $=$ multiplicity of infection

PCR $=$ polymerase chain reaction

PDGF = Platelet derived growth factor

$\mathrm{Q}-\mathrm{PCR}=$ real time quantitative reverse transcription PCR

RNAi $=$ RNA interference

siRNA $=$ small interfering RNA

VSMC = vascular smooth muscle cell

\section{Competing interests}

None declared.

\section{Authors' contributions}

Both authors participated in the design and interpretation of all the experiments presented, as well in the preparation of the manuscript. ACL performed the experiments; JJC provided overall guidance and financial support. Both authors read and approved the final manuscript.

\section{Acknowledgements}

The authors wish to acknowledge Ira Herman for providing the $\alpha$-actin antibody, and for contributing valuable discussion. We would also like to acknowledge Holly Mason for providing helpful discussion. This work was supported by NIH Grant HL49973 to JJC

\section{References}

I. Mishra-Gorur K, Delmolino LM, Castellot J.J.,Jr.: Biological functions of heparan sulfate and heparan sulfate proteoglycans. Trends Glycosci Glycotechnol 1998, 10:193-210.

2. Ayer-Lelievre C, Brigstock D, Lau L, Pennica D, Perbal B, Yeger H: Report and abstracts on the first international workshop on the CCN family of genes. Mol Pathol 200I, 54:I05-I20.

3. Delmolino LM, Stearns NA, Castellot J.J.,Jr.: COP-I, a member of the CCN family, is a heparin-induced growth arrest specific gene in vascular smooth muscle cells. J Cell Physiol 200I, 188:45-55.

4. Lake AC, Bialik A, Walsh K, Castellot J...,Jr.: CCN5 is a growth arrest-specific gene that regulates smooth muscle cell proliferation and motility. Am J Pathol 2003, 162:219-231.

5. Bork P: The modular architecture of a new family of growth regulators related to connective tissue growth factor. FEBS Lett 1993, 327:125-130.

6. Oemar BS, Luscher TF: Connective tissue growth factor. Friend or foe? Arterioscler Thromb Vasc Biol 1997, 17:1483-1489.

7. Lau LF, Lam SC: The CCN family of angiogenic regulators: the integrin connection. Exp Cell Res 1999, 248:44-57. 
8. Brigstock DR: The connective tissue growth factor/cysteinerich $6 \mathrm{I} /$ nephroblastoma overexpressed (CCN) family. Endocr $\operatorname{Rev}$ 1999, 20:189-206.

9. Perbal B: NOV (nephroblastoma overexpressed) and the CCN family of genes: structural and functional issues. $\mathrm{Mol}$ Pathol 200I, 54:57-79.

I0. Bornstein P, Sage EH: Matricellular proteins: extracellular modulators of cell function. Curr Opin Cell Biol 2002, I4:608-6I6.

II. Delmolino LM, Stearns NA, Castellot J.J.,Jr.: Heparin induces a member of the CCN family which has characteristics of a growth arrest specific gene. Mol Biol Cell 1997, 8:287a.

12. Zhang R, Averboukh L, Zhu W, Zhang H, Jo H, Dempsey PJ, Coffey $R J$, Pardee $A B$, Liang P: Identification of rCop-I, a new member of the CCN protein family, as a negative regulator for cell transformation. Mol Cell Biol I998, 18:6|3|-6|4I.

13. Pennica D, Swanson TA, Welsh JW, Roy MA, Lawrence DA, Lee J, Brush J, Taneyhill LA, Deuel B, Lew M, Watanabe C, Cohen RL, Melhem MF, Finley GG, Quirke P, Goddard AD, Hillan KJ, Gurney AL, Botstein D, Levine AJ: WISP genes are members of the connective tissue growth factor family that are up-regulated in wntI-transformed cells and aberrantly expressed in human colon tumors. Proc Natl Acad Sci U S A 1998, 95: |47|7-|4722.

14. Kumar S, Hand AT, Connor JR, Dodds RA, Ryan PJ, Trill IJ, Fisher SM, Nuttall ME, Lipshutz DB, Zou C, Hwang SM, Votta BJ, James IE, Rieman DJ, Gowen M, Lee JC: Identification and cloning of a connective tissue growth factor-like cDNA from human osteoblasts encoding a novel regulator of osteoblast functions. J Biol Chem 1999, 274: I7|23-I7I3I.

15. Champagne MJ, Dumas P, Orlov SN, Bennett MR, Hamet P, Tremblay $\mathrm{J}$ : Protection against necrosis but not apoptosis by heatstress proteins in vascular smooth muscle cells: evidence for distinct modes of cell death. Hypertension 1999, 33:906-913.

16. Clowes AW, Karnowsky MJ: Suppression by heparin of smooth muscle cell proliferation in injured arteries. Nature 1977, 265:625-626.

17. Castellot J.J.Jr., Wright TC, Karnovsky MJ: Regulation of vascular smooth muscle cell growth by heparin and heparan sulfates. [Review]. Seminars in Thrombosis \& Hemostasis 1987, I 3:489-503.

18. Majack RA, Clowes AW: Inhibition of vascular smooth muscle cell migration by heparin- like glycosaminoglycans. J Cell Physiol 1984, I I 8:253-256.

19. Lemire JM, Merrilees MJ, Braun KR, Wight TN: Overexpression of the $V 3$ variant of versican alters arterial smooth muscle cell adhesion, migration, and proliferation in vitro. J Cell Physiol 2002, 190:38-45.

20. Celentano DC, Frishman WH: Matrix metalloproteinases and coronary artery disease: a novel therapeutic target. J Clin Pharmacol 1997, 37:991-1000.

21. Pauly RR, Passaniti A, Bilato C, Monticone R, Cheng L, Papadopoulos N, Gluzband YA, Smith L, Weinstein C, Lakatta EG, .: Migration of cultured vascular smooth muscle cells through a basement membrane barrier requires type IV collagenase activity and is inhibited by cellular differentiation. Circ Res 1994, 75:4 I-54.

22. Mason DP, Kenagy RD, Hasenstab D, Bowen-Pope DF, Seifert RA, Coats S, Hawkins SM, Clowes AW: Matrix metalloproteinase-9 overexpression enhances vascular smooth muscle cell migration and alters remodeling in the injured rat carotid artery. Circ Res 1999, 85: I I79-1 I85.

23. Baker $A H$, Zaltsman $A B$, George SJ, Newby AC: Divergent effects of tissue inhibitor of metalloproteinase-1, -2 , or -3 overexpression on rat vascular smooth muscle cell invasion, proliferation, and death in vitro. TIMP-3 promotes apoptosis. J Clin Invest 1998, I01:1478-1487.

24. Hou G, Mulholland D, Gronska MA, Bendeck MP: Type VIII collagen stimulates smooth muscle cell migration and matrix metalloproteinase synthesis after arterial injury. Am J Pathol 2000, I 56:467-476.

25. Zempo N, Koyama N, Kenagy RD, Lea HJ, Clowes AW: Regulation of vascular smooth muscle cell migration and proliferation in vitro and in injured rat arteries by a synthetic matrix metalloproteinase inhibitor. Arteriosclerosis, Thrombosis \& Vascular Biology 1996, 1 6:28-33.

26. Brown DL, Hibbs MS, Kearney M, Loushin C, Isner JM: Identification of 92-kD gelatinase in human coronary atherosclerotic lesions. Association of active enzyme synthesis with unstable angina. Circulation 1995, $91: 2125-2131$.
27. Brown DL, Hibbs MS, Kearney M, Isner JM: Differential expression of 92-kDa gelatinase in primary atherosclerotic versus restenotic coronary lesions. Am J Cardiol 1997, 79:878-882.

28. Palumbo R, Gaetano C, Melillo G, Toschi E, Remuzzi A, Capogrossi $M C$ : Shear stress downregulation of platelet-derived growth factor receptor- beta and matrix metalloprotease- 2 is associated with inhibition of smooth muscle cell invasion and migration. Circulation 2000, 102:225-230.

29. Fan WH, Pech M, Karnovsky MJ: Connective tissue growth factor (CTGF) stimulates vascular smooth muscle cell growth and migration in vitro. Eur J Cell Biol 2000, 79:915-923.

30. Fan WH, Karnovsky MJ: Increased MMP-2 expression in connective tissue growth factor over- expression vascular smooth muscle cells. J Biol Chem 2002, 277:9800-9805.

31. Kondo S, Kubota S, Shimo T, Nishida T, Yosimichi G, Eguchi T, Sugahara $\mathrm{T}$, Takigawa $\mathrm{M}$ : Connective tissue growth factor increased by hypoxia may initiate angiogenesis in collaboration with matrix metalloproteinases. Carcinogenesis 2002, 23:769-776.

32. Oemar BS, Werner A, Garnier JM, Do DD, Godoy N, Nauk M, Marz W, Rupp J, Pech M, Luscher TF: Human Connective Tissue Growth Factor is expressed in Advanced Atherosclerotic Lesions. Circulation 1997, 95:83 I-839.

33. Crean JK, Finlay D, Murphy M, Moss C, Godson C, Martin F, Brady $H R$ : The role of $p 42 / 44$ MAPK and protein kinase $B$ in connective tissue growth factor induced extracellular matrix protein production, cell migration, and actin cytoskeletal rearrangement in human mesangial cells. J Biol Chem 2002, 277:44187-44194.

34. Hood JD, Cheresh DA: Role of integrins in cell invasion and migration. Nat Rev Cancer 2002, 2:91-100.

35. Soon LL, Yie TA, Shvarts A, Levine AJ, Su F, Tchou-Wong KM: Overexpression of WISP-I down-regulated motility and invasion of lung cancer cells through inhibition of Rac activation. J Biol Chem 2003, 278: I|465-I|470.

36. Castellot J.J.,Jr., Favreau LV, Karnovsky MJ, Rosenberg RD: Inhibition of vascular smooth muscle cell growth by endothelial cell-derived heparin. Possible role of a platelet endoglycosidase. J Biol Chem 1982, 257: | I 256- I I 260.

37. Castellot J.J.,Jr., Pukac LA, Caleb BL, Wright T.C.,Jr., Karnovsky MJ: Heparin selectively inhibits a protein kinase $C$-dependent mechanism of cell cycle progression in calf aortic smooth muscle cells [published erratum appears in J Cell Biol 1990 Mar; I I 0(3): 863]. J Cell Biol I 989, I 09:3 |47-3। 55.

38. Tuschl T, Zamore PD, Lehmann R, Bartel DP, Sharp PA: Targeted mRNA degradation by double-stranded RNA in vitro. Genes Dev 1999, 13:3191-3197.

39. Castellot J.J.,Jr., Cochran DL, Karnovsky MJ: Effect of heparin on vascular smooth muscle cells. I. Cell metabolism. I Cell Physiol 1985, I 24:21-28.

\section{Publish with Bio Med Central and every scientist can read your work free of charge}

"BioMed Central will be the most significant development for disseminating the results of biomedical research in our lifetime. "

Sir Paul Nurse, Cancer Research UK

Your research papers will be:

- available free of charge to the entire biomedical community

- peer reviewed and published immediately upon acceptance

- cited in PubMed and archived on PubMed Central

- yours - you keep the copyright
BioMedcentral 